\title{
Particle Swarm Optimization-Based Routing Protocol for Clustered Heterogeneous Sensor Networks with Mobile Sink
}

\author{
${ }^{1}$ Sudarmani, R. and ${ }^{2}$ K.R. Shankar Kumar \\ ${ }^{1}$ Department of Electronics and Communication Engineering, \\ Avinashilingam University for Women, Coimbatore, Tamilnadu, India \\ ${ }^{2}$ Sri Ramakrishna Engineering College, Coimbatore, Tamilnadu, India
}

Received 2012-04-15, Revised 2013-04-05; Accepted 2013-04-18

\begin{abstract}
In Wireless Sensor Networks (WSN) sensor nodes with sensing, computing and communication infrastructure are randomly deployed and organized as clusters. Most of the existing sensor networks focus on homogeneous in which the cluster heads are changed periodically. To improve the lifetime of energy constraint battery powered WSN and to avoid energy sink-hole problem; Clustered Heterogeneous Sensor Networks (HSN) are analyzed with mobile sink. Our proposed method combines load balanced clustering, transmission power control over normal nodes present in the cluster and mobile sink over HSN. PSO is used to find the optimal path for mobile sink to collect data from cluster heads. The experimental results show that the proposed system has lower energy consumption and improved lifetime over static sink, without load balancing and power control approaches. The optimal path algorithm based on PSO is more robust and easy to reach the solution for real world environmental monitoring and data aggregation problems.
\end{abstract}

Keywords: Heterogeneous Sensor Networks, Clustering, Mobile Sink, Power Control, PSO

\section{INTRODUCTION}

Wireless Sensor Networks (WSNs) consist of large number of nodes, having sensing, computation and wireless communication capabilities. WSN consist of large number of tiny sensor nodes, which are usually operated by battery and are unattended after deployment. Sensor nodes life time depends on the life time of battery power (energy). Hence battery power (energy) utilization is a critical issue in WSN. WSN is an emerging technology and have wide range of applications, such as environment monitoring, home and assisted living medical care, industrial automation, agriculture, vehicle monitoring, animal tracking, habitat monitoring and numerous military applications, (Chong and Kumar, 2003; Zhao and Guibas, 2004). They also collect information from the environment, where they deployed and reports to the remote base station.
Normally stationary sink is used in WSN and is more energy efficient when compared to the nodes present in the network. Each sensor node communicates wirelessly with a few other local nodes within its radio communication range. The existing homogeneous wireless sensor networks have sensors with equal capacity and hence they become application specific. In this study, heterogeneous sensor network is analyzed, which consists of different compositions of sensors with different capabilities such as collection of image data, collection of audio signal. The clustering method is used for communication between nodes and sink, since it is energy efficient when compare to single and multi hop routing. The cost of transmitting a bit is higher than a computation (Chong and Kumar, 2003) and hence it is advantageous to organize the sensors into clusters. In clustering, one of the sensor nodes in the cluster will be elected as Cluster Head $(\mathrm{CH})$ and which is responsible

Corresponding Author: Sudarmani, R., Department of Electronics and Communication Engineering,

Avinashilingam University for Women, Coimbatore, Tamilnadu, India Tel: 04222658145 
for relaying data from each sensor to the remote receiver. In addition, data fusion and data compression can occur in the cluster head by considering the potential correlation among data from neighboring sensors. This clustering approach is preferred because it localizes traffic and can potentially be more scalable (Bandyopadhyay and Coyle, 2003; Karl and Wilig, 2005; Santi, 2005; Wei and Chan, 2005)

In HSN there is no need of cluster head selection algorithm and network life time can be increased by reducing the energy consumption for communication and load balancing (Gupta and Younis, 2003). In the large scale HSN, as the sink is far away from the sensor nodes, each node needs more energy to send the data. The energy consumption of nodes in HSN can be reduced by introducing mobile sink. In this study, the network life time is increased by:

- Clustering of nodes

- $\quad$ Performing load balancing over clustering

- Applying adaptive transmission power control over sensors in clustered load balanced HSN

- Applying adaptive transmission power control over sensors in clustered load balanced HSN, Instead of static sink, mobile sink is introduced, which could improve coverage and localization

- $\quad$ PSO is used to determine the optimal path

\subsection{Related Work}

Wireless sensor networks have gained increasing attention from both the research community and actual users. Many clustering algorithms in various contexts have also been proposed in the past by (Baker and Ephremides, 1981; Das and Bharghavan, 1997; Lin and Gerla, 1997; Amis and Prakash, 2000; Heinzelman et al., 2000; Chiasserini et al., 2002; McDonald and Znati, 1999; Gerla and Tsai, 1995; Basagni, 1999a; 1999b; Chatterjee et al., 2002; Amis et al., 2000). Many of these algorithms aim at minimizing the energy spent in the homogeneous system.

Mhatre and Rosenberg (2004) gives guidelines about the modes of propagation, clustering and battery energy of normal and $\mathrm{CH}$ nodes. Cheng and Shi (2009) analyzed the heterogeneity with new clustering algorithm which decides the cluster head according to the node energy. $\mathrm{CH}$ selection algorithm was needed as LEACH. Hur and Kim (2008) explains about adaptive clustering and power control for homogeneous sensor networks. A survey on energy efficient scheduling mechanisms for WSN is given by (Wang and Xiao, 2005).
Yin et al. (2007) presents energy consumption analysis over clustering and it was concluded that optimum transmission range was necessary to make network active and compatible. Jayashree et al. (2006) proposed load balancing over heterogeneous sensor networks and stability of the network was analyzed.

Gao et al. (2010) have studied power control in WSN by changing the network topology to optimize network routing through adjusting transmission power. Lin et al. (2006) addresses the issue of feedback based transmission power control algorithm to dynamically maintain individual link quality over time. Kawadia and Kumar (2005) have studied power control over Ad-hoc networks.

Also most of the WSN systems adapt network level transmission power control. Most of the approaches are used for homogeneous non cluster WSN systems. In many practical applications of WSN, the mobile sink tends to move around within the sensor fields and receive data (Ye et al., 2002; Hamida and Chelius, 2008). Tracking and data delivery to sink node is discussed in Oh et al. (2010). Constructing a proper routing takes a very important role in homogeneous sensor networks, which periodically changes cluster heads. The different network topologies with mobile sink is analyzed in Yang et al. (2010).

Al-Karaki and Kamal (2004) addresses different routing techniques for WSN. Ammari and Das (2008) analyzed heterogeneity mobility and mobile sink in homogeneous using veronoi diagram. Yang et al. (2010) introduced mobile sink instead of static sink and it was compared with two different topologies.

\section{MATERIALS AND METHODS}

\subsection{Contribution and Organization}

- Energy utilization is a very critical issue in WSN. In this work, energy efficiency is obtained by considering two different sensors (Heterogeneous in terms of energy) in the network. Compared to flat and multi hop communication cluster based architecture provides long life time, hence it is preferred. In homogeneous, $\mathrm{CH}$ selection algorithm is needed to select $\mathrm{CH}$ periodically, which in turn increases the overhead. In this system $\mathrm{CH}$ are fixed

- If few $\mathrm{CH}$ nodes are heavily loaded, will consume their energy soon. To get uniform energy depletion, load balancing (equal number of nodes to each cluster) is introduced over clusters 
- To send data to the cluster heads low energy sensors (normal nodes) adjust their communication ranges according to the distance with its corresponding $\mathrm{CHs}$ (Adaptive Transmission Power is introduced)

- Frequent re-clustering, long distance communication from $\mathrm{CH}$ nodes to farthest located static sink, which in turn increase the energy consumption. To avoid energy whole problem and to prolong the network life time mobile sink is introduced. Mobile sink travels through the $\mathrm{CHs}$ and collect data from them with sojourn time. Communication is taken over single shared channel using TDMA which prevents radio interference and reduces energy consumption

- $\quad$ PSO is used to find the shortest path between the $\mathrm{CHs}$ through which sink travels, hence neighbours of sink changes which avoids energy hole as well as $\mathrm{CH}$ life time gets increased hence network life time

- Hence energy efficiency of $\mathrm{CH}$ nodes and normal nodes present in the clusters gets increased

\subsection{Network and Energy Models}

Assume uniformly deployed sensor nodes (Low energy and High energy nodes) within a LxL area with node density d. After deployment, nodes are unattended. Both the L-nodes and H-nodes are stationary and uses single hop communication to sink. The battery energy of $\mathrm{L}$ and $\mathrm{H}$ - nodes are $\mathrm{E}_{0}$ and $\mathrm{E}_{1}$ respectively. $\mathrm{H}$-nodes are less energy constraint.

\subsection{Energy Model}

Energy consumption in WSN is mainly divided into two parts, based on energy consumption for processing, computation and transmission of collected data. The energy required for data transmission will be more compared to data collection. The power dissipation of radio module is given by Equation 1and 2:

$$
\begin{aligned}
& \mathrm{E}_{\mathrm{tx}}(\mathrm{k}, \mathrm{d})=\mathrm{E}_{\mathrm{elc}}(\mathrm{k})+\mathrm{E}_{\mathrm{amp}}(\mathrm{k}, \mathrm{d}) \\
& =\mathrm{kE}_{\text {elc }}+\mathrm{k} \varepsilon_{\mathrm{fr}} \mathrm{d}^{2} \quad \mathrm{~d} \leq \mathrm{d}_{0} \\
& =\mathrm{kE}_{\text {elc }}+\mathrm{k}_{\mathrm{fr}} \mathrm{d}^{4} \quad \mathrm{~d}>\mathrm{d}_{0} \\
& \mathrm{E}_{\mathrm{rx}}(\mathrm{k})=\mathrm{kE}_{\mathrm{elc}}
\end{aligned}
$$

$\mathrm{E}_{\mathrm{elc}}$ is the electronics energy. $\mathrm{E}_{\mathrm{amp}}$ is the amplifier energy, depending on the distance to the receiver. As the distance between sources to sink plays a major role in energy consumption, the sensor nodes that transmit data over a long distance will drain energy soon. Reducing the node transmission radius will lead to less energy consumption (Mhatre and Rosenberg, 2004).

\subsection{Radio Model}

The two ray ground propagation model is used for communication. The minimum transmission power of sending node $\mathrm{P}_{\min }$ is given by Equation 3:

$\mathrm{P}_{\min }=\frac{\mathrm{P}_{\mathrm{t}} \mathrm{P}_{\mathrm{thr}}}{\mathrm{P}_{\mathrm{r}}}$

$\mathrm{P}_{\mathrm{thr}}$ is the minimal threshold power of received signal.

\subsection{Heterogeneous Sensor Network Model}

Clustering is one of the most important approaches used in WSN to save energy. Heterogeneous Sensor Network (HSN) modeled by both Low (L) as well as High $(\mathrm{H})$ Energy sensors are distributed uniformly and randomly in the environment. The powerful $\mathrm{H}$ sensors form clusters around them and act as cluster heads, since $\mathrm{CH}$ nodes are predetermined. The cluster formation is depicted in Fig. 1, consists of L sensors, $\mathrm{H}$ sensors and the Base Station (BS). H sensors provide longer transmission range, higher data rate than $\mathrm{L}$ sensors and also facilitates better protocols, algorithms and secure schemes in sensor networks.

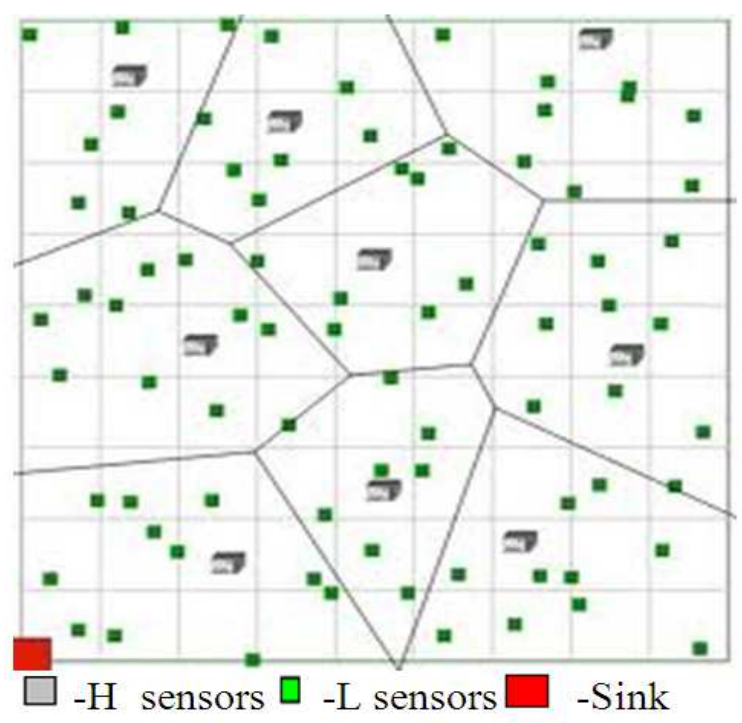

Fig. 1. Heterogeneous sensor network formation 
As an efficient and robust cluster formation scheme is adopted in HSN the sensor nodes provide coverage of the region with a high probability (Amis and Prakash, 2000). Cluster heads are responsible for data aggregation and transmission of the aggregated data to a base station.

\subsection{Energy Model of HSN}

In this model number of $\mathrm{L}$ nodes and $\mathrm{H}$ nodes are fixed that is ten percentage of population of node is act as $\mathrm{H}$ nodes and equipped with additional battery energy. $\mathrm{H}$-nodes have higher software and hardware complexity. Direct communication is taken place between L-nodes to the concerned H-node. In multi hop communication, if any node expires the network loses connectivity. But in the proposed system, if any of the nodes in the cluster dies it does not affect the network operation. The total energy consumption of heterogeneous sensor networks is obtained by combining the energy consumed by cluster heads and non cluster heads. The total energy consumed by heterogeneous sensor networks (Mhatre and Rosenberg, 2004) is given by Equation 4-12:

$$
\begin{aligned}
& \mathrm{E}_{\mathrm{T}}=\mathrm{E}_{\mathrm{HE}}+\mathrm{E}_{\mathrm{LE}} \\
& \mathrm{E}_{\mathrm{HE}}=\mathrm{T}\left(\frac{\mathrm{L}}{\mathrm{H}}\left(1_{1}+\mathrm{E}_{\mathrm{f}}\right)+\left(1_{2}+\mu_{2} \mathrm{~d}^{4}\right)\right) \\
& \mathrm{E}_{\mathrm{LE}}=\mathrm{T}\left(1_{1}+\frac{\mu_{1} \mathrm{~A}^{2}}{\mathrm{H}}\right)
\end{aligned}
$$

Where:

$\mathrm{E}_{\mathrm{f}}=$ The computational energy spent on fusion of each packet

$1_{1}=$ The amount of energy spent in the transmitter electronics circuitry within a cluster

$1_{2}=$ The amount of energy spent in the transmitter electronics circuitry from the cluster head to the base station

$\mu_{1}=$ The energy spent in the RF amplifier within the cluster

$\mu_{2}=$ The energy spent in the RF amplifier from the cluster head to the base station

$\mathrm{A}=$ The radius of the region

$\mathrm{T}=$ The data gathering cycles

$\mathrm{n}_{0} \quad=$ The number of low energy nodes

$\mathrm{n}_{1}=$ The number of high energy nodes

$\frac{\mathrm{A}}{\sqrt{\mathrm{n}_{1}}}=$ The radius of the cluster region

\subsection{Load Balancing Over Clustering}

Main objective of this study is to reduce energy consumption hence it gives increased life time. Load balanced clustering gives uniform energy depletion of all nodes present in the network by making communication with closer nodes by balancing load among the H sensors.

In a hierarchical sensor network, the $\mathrm{H}$ nodes transmit hello packets to all the nodes and the nodes in turn acknowledge (it consist of node locations) the receipt of it. Upon receipt of acknowledgment all $\mathrm{H}$ sensors compares the distance between itself to L sensors with the threshold distance. All Clusters are formed on the basis of shortest distance between $\mathrm{H}$ and $\mathrm{L}$ nodes:

$\mathrm{D}_{\mathrm{LH}}=\sqrt{\left(\mathrm{H}_{\mathrm{i}}(\mathrm{x}, \mathrm{y})-\mathrm{L}_{\mathrm{j}}(\mathrm{x}, \mathrm{y})\right)^{2}}$

where $\mathrm{i}-1,2,3, \ldots 10 \%$ of total nodes

where $\mathrm{j}-1,2,3, \ldots 90 \%$ of total nodes

$\mathrm{D}_{\mathrm{LH}}<\mathrm{D}_{\text {th }}$

It will be linked with particular $\mathrm{H}$ node else it is omitted until the counter associated with each $\mathrm{H}$ node reaches zero:

Algorithm: $\mathrm{CH}$ advertisement

H sensors announcement

L Sensors acknowledgment

Set counter value to $(\mathrm{N} / \mathrm{H})-1$ for all $\mathrm{H}$ nodes

IF $\mathrm{H}$ sensors receives ack with in a stipulated period (to avoid collisions TDMA is used)

It calculates distance between itself to all L sensors one by one and compares it with threshold distance

IF the calculated distance is less than threshold distance,

Decrement the counter value

ELSE

Do not change the counter value

IF Counter value is zero

Stop comparing

END IF

END IF

Load balanced Clusters are formed, corresponding $\mathrm{H}$ sensors will give further details to thbe corresponding members.

END IF.

Figure 2 explains about the procedure involved in getting load balancing over HSN.

\subsection{Adaptive Transmission Power Level Based Communication}

Initially all $\mathrm{L}$ nodes use the maximum transmission radius and power to communicate. All nodes in the network use its maximum communication range. 
Sudarmani, R. and K.R. Shankar Kumar / American Journal of Applied Sciences, 10 (3): 259-269, 2013

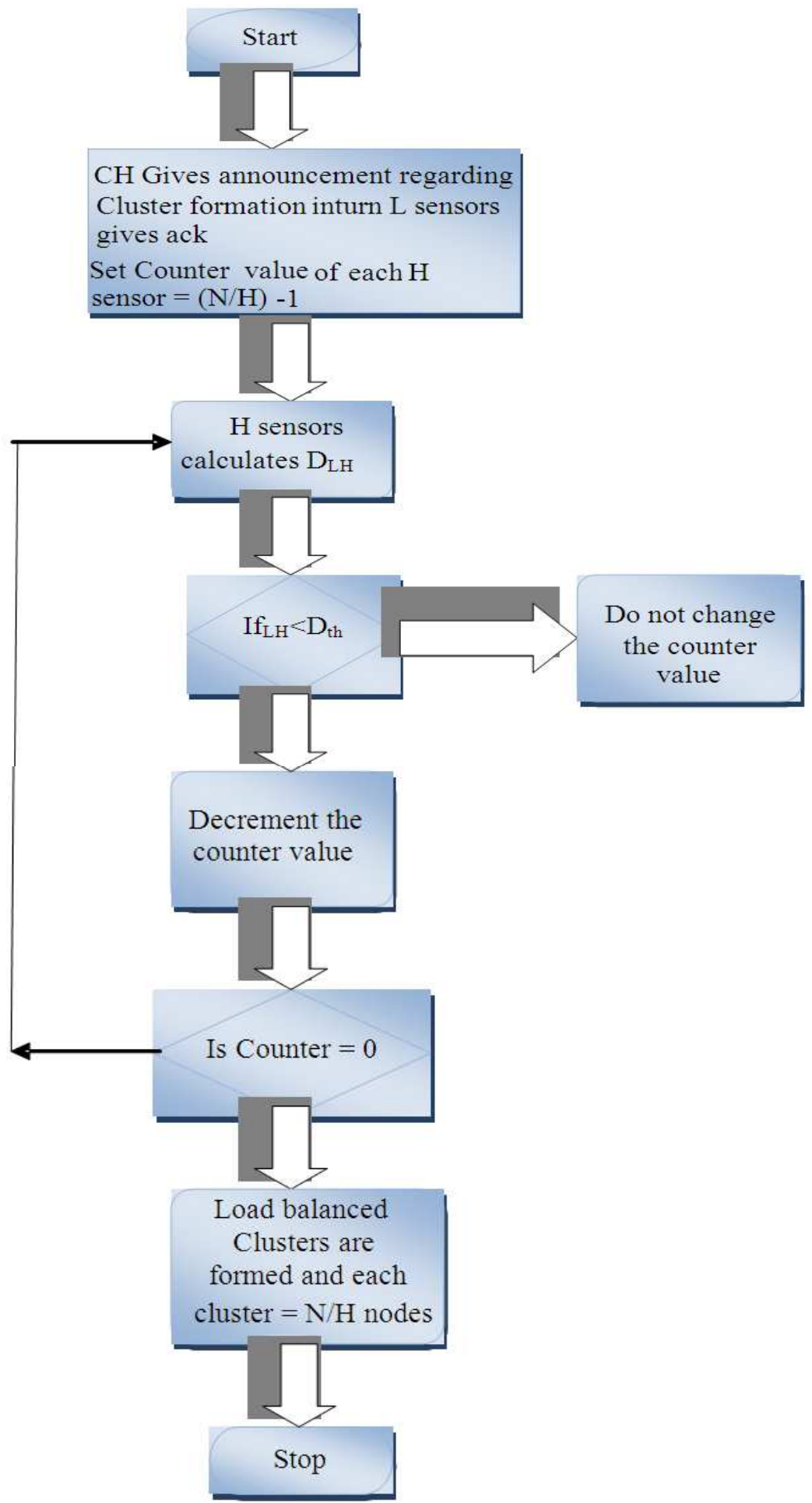

Fig. 2. Flow chart for load balancing over clustering 


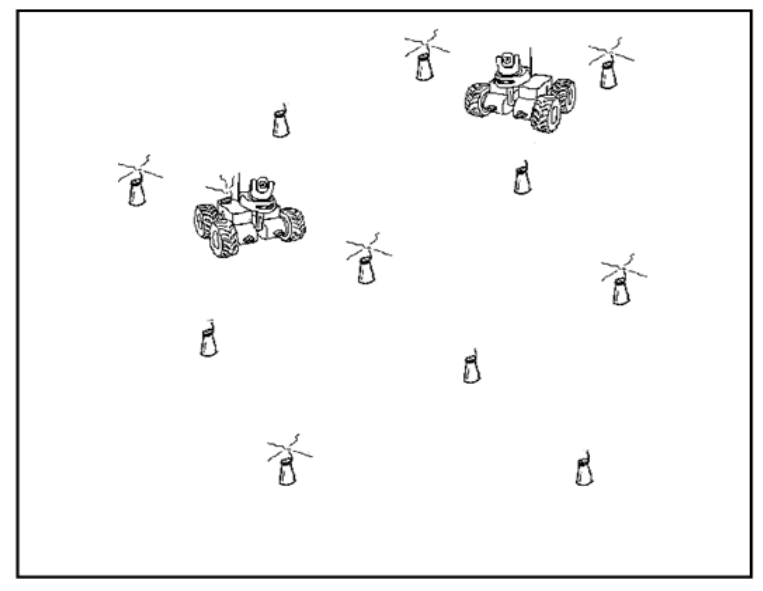

Fig. 3. HSN with mobile sink

Table 1. Various transmission ranges and corresponding power levels

\begin{tabular}{lr}
\multicolumn{2}{c}{ power levels } \\
\hline $\mathrm{Pt}(\mathrm{mW})$ & Transmission range $(\mathrm{m})$ \\
\hline 281.8 & 250 \\
225 & 200 \\
169 & 150 \\
112 & 100 \\
56 & 50 \\
28 & 25 \\
\hline
\end{tabular}

As a result of node distribution maximum transmission radius is usually longer than the distance between $\mathrm{CH}$ nodes and $\mathrm{L}$ nodes present with the clusters, which causes the waste of energy. To save energy, an L node adjusts its transmission radius to reach the corresponding $\mathrm{CH}$ alone. Initial transmission range of all nodes is to be R. After cluster formation all L sensors reduces its transmission range according to the radius of cluster it belongs to, it is to be $r$, where $r<R$.

Mapping Table 1 shows the distance between $L$ and $\mathrm{H}$ nodes and the corresponding transmission power level.

\subsection{Sink Mobility}

Sensor nodes which are far away from the sink relay their data using maximum transmission power level, hence nodes loses its energy soon. Introducing mobile sink will increase the lifetime and avoids sink whole problem, the HSN with mobile sink is shown in Fig. 3. Initially Random way point mobility model is assumed and speed of mobile sink to be ' $v$ '. Locally sensed data is buffered at each cluster and sink collects the data from the subset of nodes ( $\mathrm{CH}$ nodes) only. The aggregated data is buffered at the H-sensors until the sink enters its contact area. The procedure to collect data by mobile sink is:
- $\quad$ Sink transmits hello packets to all $\mathrm{CHs}$

- All CHs registers with sink with in a defined period

- Sink travels through the definite path calculated by shortest path routing and PSO

- When it enters the $\mathrm{CH}$ vicinity it gives beacon message

- $\mathrm{CH}$ transmits buffered data to sink after receiving message

- Sink travels and collects the data within the sojourn time

\subsection{Energy Model}

Total energy of the network is equal to sum of the energy of $\mathrm{H}$-sensors for data aggregation and transmission of the data to sink and energy spent by sink to collect data from all $\mathrm{H}$-sensors and sink movement energy. Energy of cluster head is:

$$
\begin{aligned}
& \mathrm{E}\left(\mathrm{H}_{\mathrm{i}}\right)=\text { area of each cluster } \times \text { number } \\
& \text { of nodes in the cluster }=\pi \mathrm{r}^{2} \times \frac{\mathrm{N}}{\mathrm{H}} \\
& \mathrm{S}_{\mathrm{e}}=\sum_{\mathrm{k} \in \mathrm{C}_{\mathrm{i}}} \mathrm{Tl}_{\mathrm{k}} \\
& \text { Totalenergy }=\mathrm{E}\left(\mathrm{H}_{\mathrm{i}}\right)+\mathrm{S}_{\mathrm{e}}
\end{aligned}
$$

- $l_{\mathrm{k}}$ is an integer variable which represents the number of times when the sink is located at node $\mathrm{k}, \mathrm{k} \in \mathrm{C}_{\mathrm{i}}$ at time $\mathrm{T}$

- $\mathrm{C}_{\mathrm{i}}$ is the possible set of cluster heads

- $\mathrm{T}$ is the sojourn time in which the sink has to collect data from all $\mathrm{CHs}$ and it includes the travelling and waiting time also

\subsection{Particle Swarm Optimization (PSO)}

Different routing techniques have been proposed in the literature for the mobile nodes, they are proactive, reactive and flooding schemes. In the above methods link breaks occur because of node mobility, hence route discovery becomes an energy consuming issue due to overhead. Simultaneously mobility increases more chances of energy degradation; hence efficient methods are needed for routing (Jung et al., 2011).

In this study two different routing methods have been implemented and compared in an effective manner. The methods are:

- $\quad$ Shortest path routing

- $\quad$ PSO based routing 
In the shortest path routing mobile sink finds the nearer $\mathrm{CH}$ with respect to its present position, through which it travels and collects the data from high energy nodes only.

PSO is a bio-inspired (Kennedy and Eberhart, 1995) computational method, which is a population based optimization technique which performs a parallel search on a solution space. Optimum solution is obtained from the set of randomly generated initial solutions by moving particles around in the search space, which finds the optimum solution by swarms following the best particle. Each particle has particular velocity and position, at each iteration a new velocity value is calculated and it is used to update the particle's position. The process iterates until reaching a stopping condition (optimum one).

In the classical PSO algorithm:

- Each particle has a position and a velocity

- Knows its own position and the value associated with it

- Knows the best position(pbest) it has ever achieved and the value associated with it

- Knows its neighbors, their best positions(gbest) and their values

The move of a particle is a composite of three possible choices (Onwubolu and Clerc, 2004):

- To follow its own way

- To go back to its best previous position

- To go towards its best neighbor's previous or present position

A general framework of a particle swarm optimization algorithm is given below:

Algorithm:

procedure PSO

Initialize a population of particles

Do

for each particle $\mathrm{p}$ with position $\mathrm{xp}$ do

if $\left(x_{p}\right.$ is better than pbest $\left.t_{p}\right)$ then

pbest $_{\mathrm{p}} \leftarrow \mathrm{x}_{\mathrm{p}}$

end if

end for

Define gbest $_{\mathrm{p}}$ as the best position found so far by any of p's neighbors

for each particle $\mathrm{p}$ do

$\mathrm{v}_{\mathrm{p}} \leftarrow$ Compute_velocity $\left(\mathrm{x}_{\mathrm{p}}\right.$, pbest $_{\mathrm{p}}$, gbest $\left._{\mathrm{p}}\right)$

$\mathrm{x}_{\mathrm{p}} \leftarrow$ update_position $\left(\mathrm{x}_{\mathrm{p}}, \mathrm{v}_{\mathrm{p}}\right)$

end for while (a stop criterion is not satisfied):

$$
\begin{aligned}
& \mathrm{v}_{\mathrm{p}}(\mathrm{t})=\mathrm{v}_{\mathrm{p}}(\mathrm{t}-1)+\mathrm{c}_{1} \operatorname{rand}_{1}\left(\text { pbest }_{\mathrm{p}}(\mathrm{t}-1)-\mathrm{x}_{\mathrm{p}}\right. \\
& (\mathrm{t}-1))+\mathrm{c}_{2} \operatorname{rand}_{2}\left(\operatorname{gbest}_{\mathrm{p}}(\mathrm{t}-1)-\mathrm{x}_{\mathrm{p}}(\mathrm{t}-1)\right) \\
& \mathrm{x}_{\mathrm{p}}(\mathrm{t})=\mathrm{x}_{\mathrm{p}}(\mathrm{t}-1)+\mathrm{v}_{\mathrm{p}}(\mathrm{t})
\end{aligned}
$$

The algorithm to find the shortest path has been taken from Niasar et al. (2009).

\section{RESULTS AND DISCUSSION}

\subsection{Simulation Environment}

Network simulator ns-2 is used for simulation. Two ray ground reflection model is used and 100 nodes are uniformly spread in a square region with a dimension of $200 \times 200 \mathrm{~m}$, out of which $10 \%$ are $\mathrm{H}$ nodes.parameters used in the simulation are given in Table 2. Initially less energy constraint sink is located far away from the network area.

As the residual energy of HSN with ATPC is more compared to the HSN as shown in Fig. 4, the life time of HSN with ATPC is longer compared to HSN. Simulation is carried out for calculating energy consumption by varying the number of $\mathrm{L}$ nodes. The plot of the number of $\mathrm{L}$ nodes Vs energy consumption is shown in Fig. 5. It shows that the energy consumed by HSN with ATPC is less when compared to HSN. Hence optimization of life time as well as energy consumption is achieved in the case of HSN with ATPC. Application of Load balancing leads to more residual energy than HSN with ATPC. When more packetes are transmitted the energy is saved, hence life time is also increased is shown in Fig. 6 and Fig. 7 shows the comparison graph for residual energy in HSN with mobile sink and without mobile sink.

The sink covers a distance of $685 \mathrm{~m}$ in the coverage area by the HSN nodes to cover the entire $\mathrm{H}$ nodes using shortest path method and it travels a distance of $432 \mathrm{~m}$ only using PSO (Table 3 and 4). During the sink movement the transmission energy of nodes will be minimum and the mobility factor will have very less impact because the speed of mobile sink is to be $4 \mathrm{~m}$ $\sec ^{-1}$. Thus the energy consumption by sink is minimum when compared to the static sink and it collects data from all nodes in a periodic manner.

When speed of the mobile sink increases, the round trip time gtes reduced, hence loss of data occurs. Frequent retransmission are needed by all $\mathrm{CHs}$, energy consumption gets increased. So,optimum speed is requied to collect data without loss, hence $4 \mathrm{~m} \mathrm{sec}^{-1}$ is fixed as speed of sink. 
Sudarmani, R. and K.R. Shankar Kumar / American Journal of Applied Sciences, 10 (3): 259-269, 2013

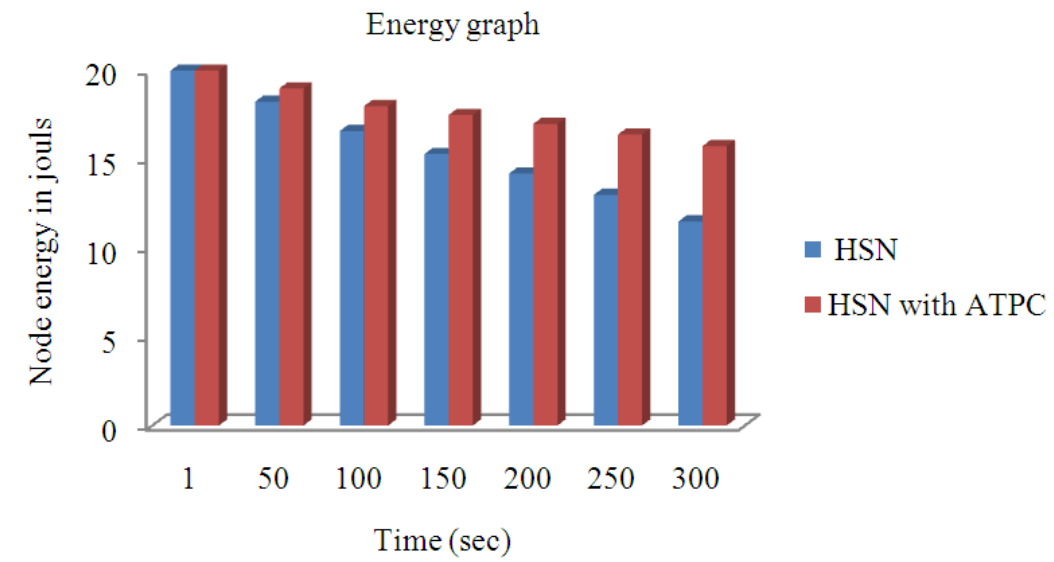

Fig. 4. Comparison graph of Energy depletion rate

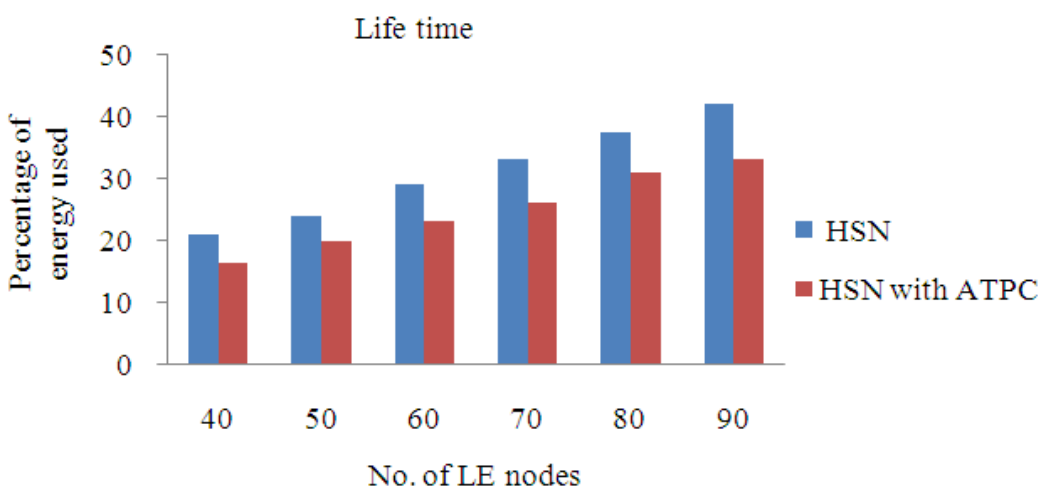

Fig. 5. Network Life time

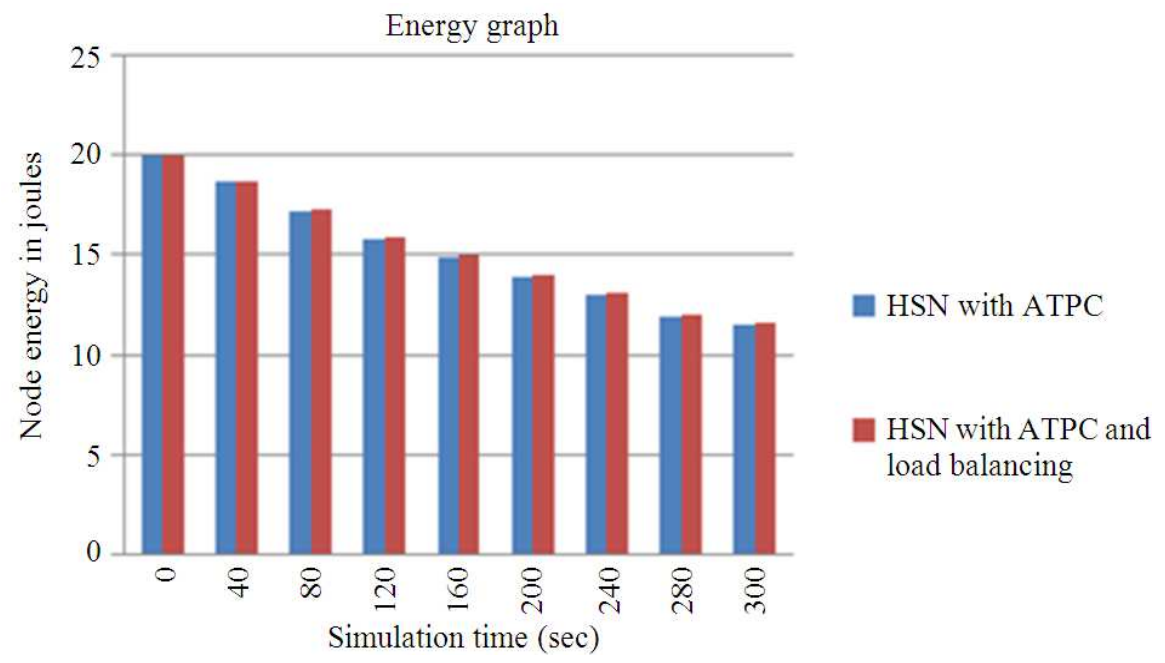

Fig. 6. Residual Energy comparison graph of HSN with ATPC and load balancing and HSN with ATPC only 


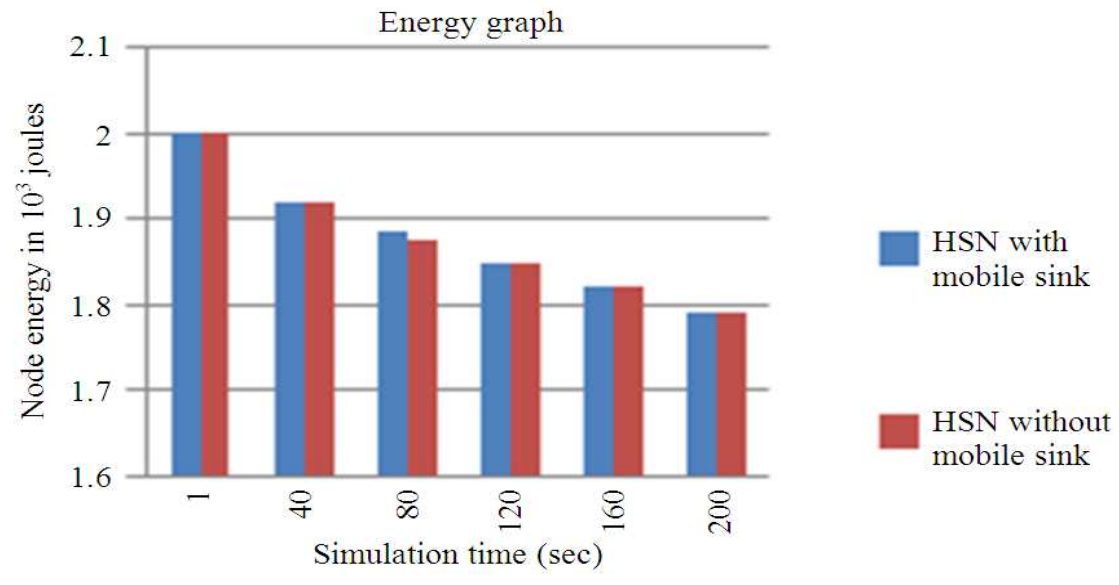

Fig. 7. Comparison graph for residual energy in HSN with mobile sink and without mobile sink

Table 2. Parameters used in the simulation

\begin{tabular}{ll}
\hline Parameter & Value \\
\hline Size of field & $200 \times 200 \mathrm{~m}^{2}$ \\
Total no. of nodes & 101 \\
Distribution type & $\begin{array}{l}\text { Random } \\
\text { distribution }\end{array}$ \\
& Two ray ground \\
Propagation model & propagation model \\
& Constant bit rate \\
Traffic load & 200 bytes \\
Length of data packet & $200 \mathrm{~m}$ \\
Initial communication Range (R) & $20 \mathrm{~J}$ \\
Initial energy of H sensors & $10 \mathrm{~J}$ \\
Initial Energy of L sensors & 0.264 \\
Energy to transmit a packet & 0.158 \\
Energy to receive a packet &
\end{tabular}

Table 3. Comparision table for travelled transmission distance by two proposed methods

\begin{tabular}{lr}
\hline Proposed Method & Transmission distance (m) \\
\hline Shortest path method & 685 \\
PSO & 432 \\
\hline
\end{tabular}

Table 4. Gives the relation between speed of mobile sink Vs time period by which it completes one trip distance

\begin{tabular}{llr}
\hline Speed $(\mathrm{m} / \mathrm{s})$ & $\begin{array}{l}\text { PSO } \\
(\text { distance in } \mathrm{m})\end{array}$ & $\begin{array}{l}\text { Shortest } \\
\text { (distance in } \mathrm{m})\end{array}$ \\
\hline 2 & 219.18460 & 342.62480 \\
4 & 109.59230 & 171.31240 \\
6 & 73.06153 & 114.20830 \\
8 & 54.79615 & 85.65620 \\
10 & 43.83692 & 68.52496 \\
\hline
\end{tabular}

\section{CONCLUSION}

Heterogeneous sensor network with ATPC is implemented and compared with heterogeneous sensor network with non ATPC for energy consumption and network life time. $\mathrm{H}$ sensors have longer transmission range, hence number of hops to reach receiver is reduced at the same time L sensors reduces its transmission range and thus energy optimization is obtained. The life time maximization is done by introducing mobile sink and it follows the optimum path which is found by PSO. The proposed method over load balanced, adaptive transmission power control HSN, the mobile sink travels a distance 1.5 times shorter than shortest path method. Hence mobile sink life time also gets increased, simultaneously HSN life time.

\section{REFERENCES}

Al-Karaki, J.N. and A.E. Kamal, 2004. Routing techniques in wireless sensor networks: A survey. IEEE Wireless Commun., 11: 6-28. DOI: 10.1109/MWC.2004.1368893

Amis, A.D. and R. Prakash, 2000. Load-balancing clusters in wireless ad hoc networks. Proceedings of the 3rd IEEE Symposium on Application-Specific Systems and Software Engineering Technology, Mar. 24-25, IEEE Xplore Press, Richardson, TX., pp: 25-32. DOI: 10.1109/ASSET.2000.888028

Amis, A.D., R. Prakash, T.H.P. Vuong and D.T. Huynh, 2000. Max-min d-cluster formation in wireless ad hoc networks. Proceedings of the IEEE, 9th Annual Joint Conference of the IEEE Computer and Communications Societies, Mar. 26-30, IEEE Xplore Press, Tel Aviv., pp: 32-41. DOI: 10.1109/INFCOM.2000.832171 
Ammari, H.M. and S.K. Das, 2008. Promoting heterogeneity, mobility and energy-aware voronoi diagram in wireless sensor networks. IEEE Trans. Parallel Distrib. Syst., 19: 995-1008. DOI: 10.1109/TPDS.2008.31

Baker, D. J. and A. Ephremides, 1981. The Architectural organization of a mobile radio network via a distributed algorithm. IEEE Trans. Commun., 29: 1694-1701. DOI: 10.1109/TCOM.1981.1094909

Bandyopadhyay, S. and E.J. Coyle, 2003. Minimizing communication costs in hierarchically clustered networks of wireless sensors. WCNC, 2: 1274-1279. DOI: 10.1109/WCNC.2003.1200556

Basagni, S., 1999a. Distributed and mobility-adaptive clustering for multimedia support in multi-hop wireless networks. Proceedings of the IEEE VTS 50th Vehicular Technology Conference, Sept. 19-22, IEEE Xplore Press, Amsterdam, pp: 889-893. DOI: 10.1109/VETECF.1999.798457

Basagni, S., 1999b. Distributed clustering for ad hoc networks. Proceedings of the 4th International Symposium on Parallel Architectures, Algorithms and Networks, Jun. 23-25, IEEE Xplore Press, Perth/Fremantle, WA., pp: 310-315. DOI: 10.1109/ISPAN.1999.778957

Chatterjee, M., S.K. Das and D. Turgut, 2002. WCA: A weighted clustering algorithm for mobile ad hoc networks. J. Cluster Comput. Special Issue Mobile Ad hoc Network., 5: 193-204.

Cheng, W. and H. Shi, 2009. AEEC: An adaptive energy efficient clustering algorithm in sensor networks. Proceedings of the 4th IEEE Conference on Industrial Electronics and Applications, May 25-27, IEEE Xplore Press, Xi'an, pp: 3950-3954. DOI: 10.1109/ICIEA.2009.5138948

Chiasserini, C.F., I. Chlamtac, P. Monti and A. Nucci, 2002. Energy efficient design of wireless ad hoc networks. Network. Technol. Services Protocols Perform. Comput. Commun. Netw. Mobile Wireless Commun., 2345: 376-386. DOI: 10.1007/3-54047906-6_30

Chong, C.Y. and S.P. Kumar, 2003. Sensor networks: Evolution, opportunities and challenges. Proc. IEEE, 91: 1247-1256. DOI: 10.1109/JPROC.2003.814918

Das, B. and V. Bharghavan, 1997. Routing in ad-hoc networks using minimum connected dominating sets. Proceedings of the IEEE International Conference on ICC 97 Montreal, Towards the Knowledge Millennium, Jun. 8-12, IEEE Xplore Press, Montreal, Que., pp: 376-380. DOI: 10.1109/ICC.1997.605303
Gao, D., L. Liang, G. Xu and S. Zhang, 2010. Power control based on routing protocol in wireless sensor networks. Proceedings of the 2nd International Conference on Future Networks, Jan. 22-24, IEEE Xplore Press, Sanya, Hainan, pp: 53-57. DOI: 10.1109/ICFN.2010.9

Gerla, M. and J.T.C. Tsai, 1995. Multicluster, mobile, multimedia radio network. J. Wireless Netw., 1: 255265. DOI: $10.1007 / \mathrm{BF} 01200845$

Gupta, G. and M. Younis, 2003. Load-balanced clustering of wireless sensor networks. Proceedings of the IEEE International Conference on Communications, May 11-15, IEEE Xplore Press, pp: 1848-1852. DOI: 10.1109/ICC.2003.1203919

Hamida, E.B. and G. Chelius, 2008. A line-based data dissemination protocol for wireless sensor networks with mobile sink. Proceedings of the IEEE International Conference on Communications, May 19-23, IEEE Xplore Press, Beijing, pp: 2201-2205. DOI: $10.1109 /$ ICC.2008.420

Heinzelman, W.R., A. Chandrakasan and H. Balakrishnan, 2000. Energy-efficient communication protocol for wireless microsensor networks. Proceedings of the 33rd Annual Hawaii International Conference on System Sciences, Jan. 4-7, IEEE Xplore Press. DOI: 10.1109/HICSS.2000.926982

Hur, H. and K. Kim, 2008. An adaptive clustering algorithm with power control in wireless sensor networks. Proceedings of the 4th International Conference on Innovations in Information Technology, Nov. 18-20, IEEE Xplore Press, Dubai, pp: 377-381. DOI: 10.1109/IIT.2007.4430499

Jayashree, L.S., S. Arumugam and N. Rajathi, 2006. E/sup 2/LBC: An energy efficient load balanced clustering technique for heterogeneous wireless sensor networks. Proceedings of the IFIP International Conference on Wireless and Optical Communications Networks, (WOCN' 06), IEEE Xplore Press, Bangalore, pp: 1-7. DOI: 10.1109/WOCN.2006.1666554

Jung, J., Y. Cho and J. Hong, 2011. Impact of mobility on routing energy consumption in mobile sensor networks. Int. J. Distrib. Sensor Netw., 2012: 12-12. DOI: $10.1155 / 2012 / 430439$

Karl, H. and A. Wilig, 2005. Protocols and Architectures for Wireless Sensor Networks. 1st Edn., John Wiley and Sons, ISBN-10: 0470095105, pp: 497.

Kawadia, V. and P.R. Kumar, 2005. Principles and protocols for power control in wireless ad hoc networks. IEEE J. Selected Areas Commun., 23: 7688. DOI: 10.1109/JSAC.2004.837354(410) 23 
Kennedy, J. and R. Eberhart, 1995. Particle swarm optimization. Proceedings., IEEE International Conference on Neural Networks, Nov. 27-Dec. 01, IEEE Xplore Press, Perth, WA., pp: 1942-1948. DOI: $10.1109 /$ ICNN.1995.488968

Lin, C.R. and M. Gerla, 1997. Adaptive clustering for mobile wireless networks. J. Selected Areas Commun., 15: 1265-1275. DOI: 10.1109/49.622910

Lin, S., J. Zhang, G. Zhou, L. Gu and John A. Stankovic et al., 2006. ATPC: Adaptive transmission power control for wireless sensor networks. Proceedings of the 4th International Conference on Embedded Networked Sensor Systems, Nov. 1-3, ACM Press, USA., pp: 223-236. DOI: 10.1145/1182807.1182830

McDonald, A.B. and T.F. Znati, 1999. A mobility-based framework for adaptive clustering in wireless ad hoc networks. IEEE J. Selected Areas Commun., 17: 1466-1487. DOI: 10.1109/49.780353

Mhatre, V. and C. Rosenberg, 2004. Design guidelines for wireless sensor networks: Communication, clustering and aggregation. Ad Hoc Netw., 2: 45-63. DOI: $10.1016 / \mathrm{S} 1570-8705(03) 00047-7$

Niasar, N.S, J. Shanbezade, M.M. Perdam and M. Mohajeri, 2009. Discrete fuzzy particle swarm optimization for solving traveling salesman problem. Proceedings of the International Conference on Information and Financial Engineering, Apr. 17-20, IEEE Xplore Press, Singapore, pp: 162-165. DOI: 10.1109/ICIFE.2009.37

Oh, S., E. Lee, S. Park, J. Jung and S.H. Kim, 2010. Communication scheme to support sink mobility in multi-hop clustered wireless sensor networks. Proceedings of the 24th IEEE International Conference on Advanced Information Networking and Applications, Apr. 20-23, IEEE Xplore Press, Perth, WA., pp: 866-872. DOI: 10.1109/AINA.2010.103

Onwubolu, G.C. and M. Clerc, 2004. Optimal path for automated drilling operations by a new heuristic approach using particle swarm optimization. Int. J. Product. Res., 42: 473-491. DOI: $10.1080 / 00207540310001614150$
Santi, P., 2005. Topology Control in Wireless Ad Hoc and Sensor Networks. 1st Edn., John Wiley and Sons, ISBN-10: 0470094532, pp: 252.

Wang, L. and Y. Xiao, 2005. Energy saving mechanisms in sensor networks. Proceedings of the 2nd International Conference on Broadband Networks, BroadNets, Oct. 3-7, IEEE Xplore Press, pp: 724732. DOI: 10.1109/ICBN.2005.1589678

Wei, D. and H.A. Chan, 2005. A Survey on cluster schemes in ad hoc wireless networks. Proceedings of the 2nd International Conference on Mobile Technology, Applications and Systems, Nov. 15-17, China, pp: 1-8. DOI: 10.1049/cp:20051497

Yang, T., M. Ikeda, G. Mino, L. Barolli and A. Durresi et al., 2010. Performance evaluation of wireless sensor networks for mobile sink considering consumed energy metric. Proceedings of the IEEE 24th International Conference on Advanced Information Networking and Applications Workshops, Apr. 20-23, IEEE Xplore Press, Perth, WA., pp: 245-250. DOI: 10.1109/WAINA.2010.50

Ye, F., H. Luo, J. Cheng, S. Lu and L. Zhang, 2002. A Two-tier data dissemination model for large-scale wireless sensor networks. Proceedings of the 8th Annual International Conference on Mobile Computing and Networking, Sep. 23-28, ACM Press, USA., pp: 148-159. DOI: 10.1145/570645.570664

Yin, B., H. Shi and Y. Shang, 2007. Analysis of energy consumption in clustered wireless sensor networks. Proceedings of the 2nd International Symposium on Wireless Pervasive Computing, Feb. 5-7, IOEEE Xplore Press, San Juan. DOI: 10.1109/ISWPC.2007.342577

Zhao, F. and L.J. Guibas, 2004. Wireless Sensor Networks: An Information Processing Approach. 1st Edn., Morgan Kaufmann publishers, San Francisco, ISBN-10: 9781558609143, pp: 358. 